\title{
Stefan Marinov's seasonal puzzle
}

With yet another holiday season brewing up in the Northern Hemispehere, readers may wish to brood on a conundrum devised by an anti-relativist. No prizes are offered for a solution.

STEFAN Marinov, the exiled Bulgarian physicist now living in Graz in Austria, is himself a puzzle. He is indefatigable in the prosecution of what seems to be his only cause, which is to prove that Einstein's theory of relativity is a pack of lies.

Marinov claims, among other things, to have shown by direct measurement that the velocity of light differs according to the direction of its travel along a fixed path. He has also partly developed what he says is a perpetual motion machine, a kind of Wimshurst machine driven by an electric motor which, when last inspected at this journal's London office, was said to require a car battery for its operation.

But most of Marinov's work is theoretical. Over the years, he has bombarded this and other journals with a series of papers with titles such as "The myths in physics" and "Violations of the laws of conservation of angular momentum and energy". Details of his correspondence with several editors may be found in his series of volumes called The Thorny Way of Truth, of which seven volumes have now been published by International Publishers (East-West) at Graz, of which Marinov appears to be the sole owner.

(From time to time, Marinov threatens to immolate himself in front of a British embassy or consulate if he cannot get an answer to a question about the publication of one of his papers. Some years ago, when embarked on such an enterprise outside the British consulate at Genoa, Marinov ran away when the police approached. Afterwards he explained that he had done so because he was in Italy illegally, without a visa. Mercifully, he has not threatened to immolate himself on Nature's account for many months.)

Part of everybody's difficulty is that too little is said about the details of the experiments whose results are said to contradict common expectation for even sympathetic critics to be able to provide constructive statements of how they and Marinov part company. Another is that Marinov's single-minded zeal for his own conviction considerably outstrips the zeal of those who may disagree. But from time to time, in Marinov's copious writings, there are relatively simple arguments that appear accessible even to those still at high school. Here is one series of gedanken experiments presented as if it were a Christmas puzzle (the original intention), with some helpful (or misleading) hints for its solution.

The figure shows a pair of circular con- ductors arranged as two concentric circles. Equal electrical currents are circulated in each, but in opposite directions. The simplest way of creating this arrangement is to cut through the concentric pair at some point and to join the loose ends in pairs by short lengths of straight conductor. An electromotive force applied anywhere along the conductor will engender a current which must be everywhere uniform. At the bridged gap, there will be equal currents flowing in opposite directions, so their influence on the magnetic fields in the concentric gap will be zero.

The device is thus a means of arranging that there is a uniform magnetic field in the space between the concentric circles in a direction perpendicular to their plane (downwards into the plane of the paper when the current in the circuit flows in the direction indicated). The sensor in the experiment is a conductor long enough just to bridge the gap between the concentric circles and mounted on thin insulating supports in such a way that it can be made to slide around the circle. The objective is to measure the voltage across the sliding conductor, either by a standard voltmeter or by a condenser whose accumulated charge will be a measure of the voltage in a steady state.

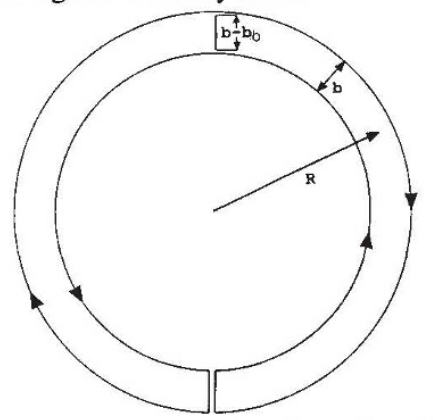

The simplest case is when the sliding conductor is at rest. Then there is no voltage. Right? Next comes the case in which the sliding conductor moves at uniform speed around the concentric gap, always pointing along a radius of the concentric circles. As the slider moves, it will cut. through magnetic lines of force at a constant rate, so there will be a constant voltage across the ends. The polarity of the slider will depend only on the direction of the current in the concentric circuit, and not on whether the slider moves clockwise or anticlockwise. Right again?

Now comes the tricky part, at least so far as Marinov is concerned. What happens if the sliding conductor is fixed in space, but the underlying concentric cir- cuit is rotated about its centre? Relativity theory naturally predicts that the voltage across the sliding conductor would be the same as in the first experiment, and with the same polarity. On the other hand, questions may be raised about the degree to which the pattern of magnetic forces generated by the current is dragged around the ring by its rotation. Maybe there is a smaller voltage, but with the same polarity. What, asks Marinov, is the answer?

The second conundrum is superficially simpler: simply rotate the apparatus in its own plane, about the centre of the concentric circles. (There will be a small voltage due to the Earth's magnetic field, but this may safely be neglected.) Is there now a voltage, and with what polarity? If the answer to the first question is "Yes" the answer to the second must be "No", and vice versa. Readers are invited to make up their minds before reading on.

Marinov's own answers are unambiguous. Vice versa wins the day. When the underlying concentric circuit is rotated and the slider is kept fixed, there is no voltage across the movable conductor. But when the whole apparatus is rotated about its centre, the voltage across the now-moving sliding conductor is identical with that obtained when the slider is moving relative to the concentric circuit.

The implications are evidently important. The null answer to Marinov's first question implies that relativity has vanished through the window, the affirmative answer to the second implies that an isolated apparatus carrying a circulating current will generate a voltage when rotated, which raises forbidden questions about absolute space. Indeed, Marinov has devised a gedanken measurement of the Earth's velocity through space by stretching his concentric circuit into a linear version of it. He also claims that these violations of simple expectation are the basis on which his perpetual motion machine is built.

What is the truth? Nobody is quite sure, for nobody has done the experiments not even Marinov. Indeed, one's confidence in the whole enterprise is somewhat undermined by Marinov's flat statement that he does not bother to repeat experiments whose outcome must be obvious. For one whose confidence in his own heterodoxy appears to be sustained only by his confidence that experiments will prove him right, Marinov seems curiously passive in the business. John Maddox 